\title{
Aging-related microstructural alterations along the length of the cingulum
} bundle

Sibilia, Francesca; Kehoe, Elizabeth Grace; Farrell, Dervla; Kerskens, Christian; O'Neill, Desmond; McNulty, Jonathan Paul; Mullins, Paul Gerald; Bokde, Arun

\section{Brain connectivity}

DOI:

10.1089/brain.2017.0493

Published: 01/08/2017

Peer reviewed version

Cyswllt i'r cyhoeddiad / Link to publication

Dyfyniad o'r fersiwn a gyhoeddwyd / Citation for published version (APA):

Sibilia, F., Kehoe, E. G., Farrell, D., Kerskens, C., O'Neill, D., McNulty, J. P., Mullins, P. G., \& Bokde, A. (2017). Aging-related microstructural alterations along the length of the cingulum bundle. Brain connectivity, 7(6), 366-372. https://doi.org/10.1089/brain.2017.0493

\footnotetext{
Hawliau Cyffredinol / General rights

Copyright and moral rights for the publications made accessible in the public portal are retained by the authors and/or other copyright owners and it is a condition of accessing publications that users recognise and abide by the legal requirements associated with these rights. study or research.

dhe public portal for the purpose of private

- You may not further distribute the material or use it for any profit-making activity or commercial gain

- You may freely distribute the URL identifying the publication in the public portal ?
}

Take down policy

If you believe that this document breaches copyright please contact us providing details, and we will remove access to the work immediately and investigate your claim. 


\section{Aging-related microstructural alterations along the length of the cingulum}

\section{bundle}

Francesca Sibilia $^{1 *}$, MSc, Elisabeth G. Kehoe ${ }^{1}, \mathrm{PhD}$, Dervla Farrell', BA, Christian Kerskens ${ }^{2}$, PhD, Desmond O'Neill ${ }^{2,3}, \mathrm{MD}, \mathrm{FRCPI}$, Jonathan P. McNulty ${ }^{4}$, PhD, Paul Mullins ${ }^{5}$, PhD , Arun L.W. Bokde ${ }^{1}$, PhD

1.Cognitive System Group, Discipline of Psychiatry, School of Medicine and the Trinity College Institute of Neuroscience (TCIN), Trinity College Dublin, Dublin, Ireland.

2. Trinity College Institute of Neuroscience, Dublin, Ireland

3. Center for Ageing, Neuroscience and the Humanities, Trinity Center for Health Sciences, Tallaght Hospital, Dublin, Ireland.

4. Radiography and Diagnostic Imaging, School of Medicine, University College Dublin, Dublin, Ireland

5.Bangor Imaging Center, School of Psychology, Bangor University, UK.

\section{"Corresponding Author:}

Francesca Sibilia

Lloyd Institute, Trinity College Institute of Neuroscience, Trinity College Dublin, Dublin 2, Ireland.

Email address: $\underline{\text { fsibilia@tcd.ie }}$

Tel: $+353-(0) 18964102$

Words: 3158

Keywords: aging, white matter, cingulum, diffusion tensor imaging (DTI), tractography, CSD-tractography 


\section{Abstract}

The aim of this study was to investigate the aging-related structural changes of the cingulum, one of the major components of the limbic network, which has a critical role in emotion, attention and memory. 35 healthy young adults $(22.3 \pm 2.7$ years) and 33 healthy older adults (69.5 \pm 3.5 years) were recruited. Diffusion weighted imaging data were acquired with a b-value $=2000 \mathrm{~s} / \mathrm{mm}^{2}$ and 61 diffusion directions and 4 non-weighted images. The fiber directions in each voxel were based on the constrained spherical deconvolution (CSD) model. The cingulum was segmented into three branches using deterministic tractography (subgenual, retrosplenial and parahippocampal), using a region-of-interest (ROI)-based approach. Atlas-based tractography $(\mathrm{ABT})$ was the method used to obtain the output tracts of each branch of the cingulum. Along-tract analysis was performed on each branch. We found a statistically significant change with aging in the left subgenual branch of the cingulum with a decrease in fractional anisotropy (FA) and axial diffusivity (AD), as well as an increase in radial diffusivity (RD). No statistically significant differences were found between young and older groups in the other two branches. This study adds to knowledge about how the cingulum changes structurally along its entire length during aging in a more detailed way, thanks to an advanced methodological approach. 


\section{Abbreviations}

CSD: Constrained Spherical Deconvolution

FA: Fractional Anisotropy

MD: Mean Diffusivity

AD: Axial diffusivity

RD: Radial Diffusivity

DWI: Diffusion Weighted Imaging

DTI: Diffusor Tensor Imaging

WM: White Matter

CB: Cingulum bundle

BDI II: Beck's Depression Inventory II

CERAD: Consortium to Establish a Registry for Alzheimer's Disease

MMSE: Mini Mental State Examination

NART: National Adult Reading Test

SLF: Superior Longitudinal Fasciculus

PRM: Pattern Recognition Memory 


\section{Introduction}

During the lifespan, the brain is subject to aging-related changes in volume, metabolism and cognition. White matter (WM) volume changes with an inverted $U$ shape in relation to aging, increasing until age 40s (Peters, 2006), followed by a plateau phase till age 60s, and start declining in older ages. Myelin sheaths deterioration leads to neural loss, decrease of fiber number and reduction of WM volume (Gunbey, et al., 2014), causing alterations in brain connections which underlie cognitive abilities.

The cingulum bundle $(\mathrm{CB})$ is one of the most prominent fiber bundles in the brain, going from the anterior to the posterior part of the brain (Jones, et al., 2013) . It has short and long associative fibers populations, which connect different brain areas, including frontal, parietal and temporal regions (Catheline, et al., 2010); functionally, it is involved in attention, memory, problem solving, visual and spatial abilities, as well as in regulating heart rate and blood pressure (Gunbey, et al., 2014).

Previous studies have measured the changes in the CB integrity during aging, analysing the average value of the entire tract, not focusing on changes along the tract length (Jang, et al., 2016,Sullivan and Pfefferbaum, 2006). Sullivan and Pfefferbaum(2006) proposed WM tracts integrity started decreasing in frontal circuits, describing an anteroposterior gradient, similar to the pattern of cognitive declines found in aging. Jang's study results (2016) supported this hypothesis; the authors divided the cingulum into five parts, and observed a WM integrity decrease first in the anterior fiber branches.

Literature about WM changes during normal aging is not homogeneous, but a few studies reported no significant differences along the cingulum with healthy aging 
(Gunbey, et al., 2014,Stadlbauer, et al., 2008). Stadlbauer et al. (2008) did not find any significant age-related modifications in the cingulum, either in the diffusivity parameters or in the number of fibers per voxel. Furthermore, Gunbey and colleagues (2014) subdivided the fornix, the cingulum and the parahippocampal gyrus, finding no FA changes in older healthy adults compared to younger people.

The studies mentioned above used a DTI-based approach, which presents limitations in brain areas with complex WM frameworks. In the present study, a constrained spherical deconvolution (CSD)-approach tractography (Jeurissen, et al., 2011) was chosen, as it allows a more accurate reconstruction of fiber pathways and gives a higher sensitivity in identifying microstructural anomalies (Reijmer, et al., 2012).

The main hypothesis of this study was that aging lead to decreased structural integrity of the cingulum compared to healthy young group. The cingulum was divided in three segments, based on a ROI-based methodological approach (Jones, et al., 2013). The methodological novelty of this study was the along-tract analysis for each branch of the cingulum to locate the structural changes with aging, as well as segmenting each individual branch.

\section{Material and Methods}

\subsection{Participants}

35 healthy young adults $(22.3 \pm 2.7$ years) and 33 healthy older people $(69.5 \pm 3.5$ years) were recruited through online advertisement. Demographic information is presented in Table 1. Each participant went through neuropsychological tests and an 
MRI scan. The study had approval from the Research Ethics Committee of the Adelaide and Meath Hospital, incorporating the National Children's Hospital, and St. James Hospital. All participants gave written informed consent before taking part in the study.

\subsection{Neuropsychological Testing}

The neuropsychological tests that participants underwent were the Beck's Depression Inventory II (BDI-II) (Beck et al, 1996) and the NART (National Adult Reading Test, Nelson et al., 1982); the older group of participants underwent the CERAD (Consortium to Establish a Registry for Alzheimer's Disease (Morris, et al., 1988) and the MMSE (Mini Mental State Examination (Folstein, et al., 1975), in order to exclude any possible cognitive impairment. The scores in each sub-test of the CERAD were within 1.5 standard deviation of the age-corrected norms, and the score in the BDI were below 14.

Both groups performed the CANTAB (Cambridge Neuropsychological Test Automated Battery (Sahakian, et al., 1988)), a computerized group of cognitive tests. Three different tests from the CANTAB were included: (a) learning of abstract images (immediate recognition and delayed recognition tests), (b) paired associate learning (PAL), and (c) spatial working memory (SWM). The learning task used was the Pattern Recognition Memory (PRM), which is a test of visual pattern recognition memory in a 2-choice forced discrimination paradigm. The PAL assesses episodic memory and new learning; it has twenty-one outcome measures, covering the errors made by the participant, the number of trials required to locate the pattern(s) correctly, memory scores and stages completed. Lastly, the spatial working memory 
task had different levels of difficulty. The scores were normalized accounting for age and gender of the participant and included in the analysis (Table 2 includes group results): PRM initial standardized ,PRM delay standardized, PAL Total Error Standard, PAL Total Error 6 Standardized (with errors recorded when there are 6 different patterns), SWM between-errors standardized and SWM Strategy Standardized.

\subsection{MRI scanning protocol}

Scanning was performed on a 3.0 Tesla Philips Achieva system (Best, The Netherlands) equipped with an eight channel head coil. The diffusion weighted imaging (DWI) sequence was a whole-brain high angular resolution diffusion imaging (HARDI) acquired with a TR $=14,556 \mathrm{~ms}$ and $\mathrm{TE}=81 \mathrm{~ms}$. A parallel sensitivity

encoding (SENSE) approach (Pruessmann, et al., 1999) with a reduction factor of 2 was used during the DWI acquisition. Single-shot spin echo-planar imaging (EPI) was used to acquire the DWI data with following parameters: matrix $112 \times 112$, isotropic voxel of $2 \times 2 \times 2 \mathrm{~mm}^{3}$ and 60 slices, with $2 \mathrm{~mm}$ thickness, and no gap between the slices. Diffusion gradients were applied in 61 isotropically distributed orientations with $b=2000 \mathrm{~s} / \mathrm{mm}^{2}$, and four images with $\mathrm{b}=0 \mathrm{~s} / \mathrm{mm}^{2}$ were acquired.

\subsection{DTI pre-processing}

The raw data were par/rec format that were converted to NIFTI format (using Chris Rorden's "dcm2nii" tool, and then read into ExploreDTI v4.8.4 (Leemans and Jeurissen, 2009), the software used for the preprocessing and analysis. Data quality 
control was assessed by checking for motion artifacts and image orientation; DWI images color maps and gradient components were also checked. Correction for head motion, eddy current (EC) and EPI distortion was performed thereafter. The data were corrected for distortions and head motion using an affine transformation to the non-diffusion-weighted images (Leemans and Jones, 2009). For EPI susceptibility correction the $\mathrm{T} 1$-weighted anatomical image was co-registered to the diffusion image.

\subsection{Tractography of the cingulum}

The cingulum segmentation was based on CSD-based (Jeurissen, et al., 2011) method. This approach has been chosen over a DTI-based one because it takes into account complex white matter orientation (Reijmer, et al., 2012). After the preprocessing, a CSD-whole brain tractography was performed. Each trajectory was followed until the direction of the pathway changed with an angle of more than $60^{\circ}$. In this study, the cingulum was divided in three segments, namely subgenual, retrosplenial and parahippocampal branches, in both hemispheres. The segmentation of each tract was performed following an established method (Jones, et al., 2013), with a modification in segmenting the parahippocampal subdivision, developed in our lab. Regions of interest (ROIs) were drawn on the subjects' native space to define each segment in the most accurate way. To get the fibers branches from each participant, an atlas-based tractography (ABT) approach was performed, based on the idea of drawing ROls on a template and applying them on other participants' images after co-registration (Lebel, et al., 2008). Two templates were picked to account for the anatomical variability in the two groups and to get an improved segmentation of the cingulum. It should be emphasized that the location of 
the ROls were not different relative to the anatomical landmarks used to place them (described below).

\subsection{Definition of ROls}

Figure 1 illustrates the location of the ROls to segment the three branches of the cingulum (Jones, et al., 2013). The first AND ROI of the subgenual section was drawn five slices anterior the back of the curve of the genu and the second AND ROI was placed on the third/fourth coronal slice caudal to the most anterior part of the genu. A NOT gate was added on the same slice of the first AND gate. The retrosplenial branch was defined placing the first AND ROI five slices posterior the front of splenium, while the second AND gate was determined by finding the most ventral plane of splenium and identifying the horizontal section, that was three or four slices above the base of splenium (Jones, et al., 2013). The parahippocampal branch was segmented by drawing the first AND gate in the same location as the second AND ROI in the retrosplenial tract; the second AND ROI was placed in the posterior part of the hippocampus. The first NOT gate was positioned above the body of the corpus callosum (Jones, et al., 2013). A second NOT gate was placed 17 slices $(\sim 25 \mathrm{~mm})$ posterior to the first one, to isolate the tract better. After performing ABT, output tracts from ExploreDTI were finally defined using additional NOT gates where necessary.

\subsection{Statistical Analysis}

Data statistical analysis was performed using SPSS (SPSS Software 22, IBM). The variables were the DTI indexes, namely FA, MD, axial and radial diffusivity $(A D$ and 
RD). The FA and diffusivity measures were analyzed by an along-tract analysis, which makes possible to determine WM structural variation along each point of the cingulum. It is an approach developed by Colby and colleagues (Colby, et al., 2012), detecting within-tract heterogeneities. The right and the left hemisphere were considered separately. The three fiber bundles were sampled within ExploreDTI, to have the same number of points spread along the length in each participant. The number of points was calculated dividing the average -across subjects- of the tract length and the voxel size (Colby, et al., 2012). The left subgenual was divided in 54 points, the left retrosplenial presented 51 points and the left parahippocampal was split in 35 points. In the right hemisphere, the subgenual part was divided into 53 points, the retrosplenial in 49 points and the parahippocampal branch in 37 points. Each fiber bundle (i.e. set of trajectories) was reduced to a single "averaged" pathway and the DTI-based measures (FA, MD, RD, AD) were extracted and analysed in a repeated-measures ANOVA model. The p-value chosen was $\mathrm{p}=0.0027$, which is a global $\mathrm{p}<0.05$ after Bonferroni correction for multiple comparisons (Nichols and Hayasaka, 2003), that is divided by the three diffusivity directions, the number of branches and the number of hemispheres considered. False discovery rate (FDR) correction was used in the along-tract analysis to correct for multiple comparisons following the Benjamini and Hochberg FDR procedure (Benjamini and Hochberg, 1995) (http://imaging.mrccbu.cam.ac.uk/statswiki/FAQ/FDR). Independent t-tests were used to localize the points that resulted statistically significant after the repeated-measures ANOVA.

Furthermore, an intra-group linear correlation between the DTI parameters and the CANTAB tests was also carried out for each of the three branches. 


\section{Results}

\subsection{Tractography Analysis}

A between-group (healthy young and older) effect was observed in the subgenual part of cingulum in the left hemisphere. Results show a statistically significant decrease in FA ( $p=0.0013$, FDR corrected) and $A D(p=0.0024$, FDR corrected) in the rostral part of the subgenual branch, as well as a statistically significant increase of $\mathrm{RD}(\mathrm{p}=0.0011$, FDR corrected) in the caudal part of the subgenual branch of the healthy older people (Figure 3).

3.2. Within-group linear correlation. A within-group linear correlation was conducted between each of the DTI measures and the scores of each test of the CANTAB, both with averaged diffusivity and along-tract diffusivity measures. There were no statistically significant linear correlations detected between any averaged measures and cognitive tests, while the results of the linear correlation with the along-tract measure showed linear correlation in the right hemisphere, but did not survive the FDR correction.

\section{Discussion}

The current study advanced our knowledge about aging-related changes by employing a CSD-approach to segment the cingulum branches; it analysed the fiber integrity measures along the tract length. Previous studies revealed that tractaverage diffusivity measures change in the older participants represented by a decrease in $F A$ and $A D$ and an increase in $\mathrm{MD}$ and $\mathrm{RD}$ compared to a healthy young 
group (Catheline, et al., 2010,Jang, et al., 2016,Sullivan and Pfefferbaum, 2006). We performed a group comparison approach to determine whether there were changes in diffusivity measures during aging, but our analysis didn't include the measurement of the change magnitude; this could be a step to consider in a further analysis.

Our findings were consistent with the hypothesized anterior-posterior gradient pattern in WM demyelination with aging (Madden, et al., 2012,Sullivan and Pfefferbaum, 2006), and consistent with other WM studies using a DTI-based approach (Head, et al., 2004,Pfefferbaum, et al., 2005). We found a high similarity with the results of Catheline et al (Catheline, et al., 2010). They found a decrease in the FA of the left subgenual between healthy young and older adults, while there was no significant difference in the other branches. Differently from Catheline et al., we measured changes not only in FA, but also in the other diffusivity measures, finding a decrease in $A D$ and increase in $R D$ of the left hemisphere.

The subgenual branch showed a decrease of FA and $A D$ in the rostral part of the branch, and an increase of RD in the posterior end. Before the FDR correction, diffusivity measures changes were seen in the same points of the branch for FA, AD and $\mathrm{RD}$, but after the correction, $\mathrm{RD}$ increased only in the posterior end of the branch. The relation between cognitive changes and the cingulum points showing significant differences is still unknown, even though a previous study (AbdulRahman, et al., 2011) on diffusion measures changes in schizophrenia patients found an increase of RD in the same points, suggesting a demyelination process and disrupt of connectivity. Bennett and colleagues (Bennett et al., 2010) explained such variation of $\mathrm{DTI}$ measures (FA/AD decrease and RD increase) to be associated with an underlying lesion-induced axonal loss and gliosis, found already in the internal 
capsule, the superior corona radiate and the frontal forceps, but it is the first time that is detected in the subgenual cingulum.

The subgenual branch is involved in selective attention, emotional conflict and perceptual processing (Jang, et al., 2016). Madden and colleagues (Madden, et al., 2012) reviewed DTI studies of the WM integrity, considering the relation between DTI measures, neurobiological changes and decline of cognitive performances in WM structures of older people, including the cingulum. They pointed out that sensory and motor functions (defined fluid cognitive abilities), that are associated with the anterior cingulate, tend to decline with aging, reflecting changes in the integrity of the WM tracts, while the crystallized abilities (referred to expertise and knowledge) remain quite stable.

Older people are able to compensate for a decrease in the WM density and networks efficiency occurring in aging, as explained by Park and Reuter-Lorenz. They described studies on functional neuroimaging and aging show how old people can perform the same task as younger people, in spite of their neural decline. This is possible when, in older people, both hemispheres are activated in cognitive tasks that require only one hemisphere in younger people (Park and Reuter-Lorenz, 2009).

Our study supports the idea that aging-related changes are not homogenous across the length of the subgenual branch; this study gives more specific insights on the exact locations of the changes along the bundle, previously demonstrated only in clinical populations (Abdul-Rahman, et al., 2011). In the older group, significant differences were seen in the rostral part of the branch for AD and FA, specifically at the point of the major curve of the segment, corresponding to the rostral part of Brodmann area 32. A study about the parcellation of the cingulate cortex 
(Beckmann, et al., 2009) suggested the locations of the differences found in the subgenual branch correspond to the area where the subgenual cingulum is connected to the orbitofrontal and medial prefrontal cortex, confirmed by restingstate functional connectivity studies (Cao, et al., 2014). RD showed statistically significant changes only in the caudal part, where the fibers exit the cingulum bundle and reach the precuneus, as seen in primates (Heilbronner and Haber, 2014).

The observed discrepancies between studies may be due to methodological differences in anatomical definitions of the cingulum regions, as well as to different $b$ values. DTI studies usually have a b-value between 500 and $1200 \mathrm{~s} / \mathrm{mm}^{2}$, whereas our study had a b-value of $2000 \mathrm{~s} / \mathrm{mm}^{2}$. The images with a higher b-value appear "noisier" when compared with images with a lower b-value, given by a larger signalto-noise ratio (SNR) (Burdette, et al., 2001). The choice of a higher b-value is because we wanted to utilize a CSD-type approach to segment the cingulum (Jeurissen, et al., 2011). The CSD approach has significant advantages over a DTIbased approach, primarily a more accurate segmentation of the $\mathrm{CB}$, and the reconstruction of the original fiber orientation, without any a priori assumption about the number of fiber populations, as required in the DTI-model (Tournier, et al., 2004). The $\mathrm{CB}$ is one of the biggest fiber bundles in the brain, presenting several fiber populations along its length; the DTI-model can create artefacts when there are more than two different fiber populations in the same voxel, whereas the CSD-approach reconstructs the fiber orientation with more than two fiber population and solve for the intra-voxel fiber orientation (Jeurissen, et al., 2011). Finally, one of the CSDapproach assumptions is that the response function seen for a certain fiber population orientation is constant throughout the brain. This may change in WM structures where there are different diffusion characteristics, for example in fibers 
with different myelination levels or axonal densities. In these cases, the response function would change the anisotropy of a small percentage, but the results would be still reliable and the fiber orientation would be still preserved (Tournier, et al., 2004).

In our study variability in regional anatomy influenced the choice of the templates required to obtain robust tracts. The atlas-based approach was chosen over the manual segmentation to minimize the differences in ROls size and location that could arise with manual drawing (Pievani, et al., 2010). A first attempt was made using one template for both groups, but the lateral ventricles atrophy due to aging led to inaccuracies in the segmentation of the tracts, because the ROls were located incorrectly relative to the enlarged ventricles (Figure 1; d) and e)). Thus the large anatomical changes due to aging necessitated the use of two templates to correctly place the ROls, one template with small lateral ventricles and another template with enlarged lateral ventricles typically seen in older populations.

Within-group linear correlations were performed between CANTAB scores and each branch of the cingulum, to test if there was a linear association between memory performance and the fiber microstructure. We did not detect any statistically significant association between memory performance and cingulum integrity in either group.

\section{Conclusion}

In the present study, the three branches of the cingulum were investigated using constrained spherical deconvolution that allowed for detailed analysis along the cingulum. The findings of this study add to our knowledge about the brain during 
aging, and it helps in further elucidating the changes in aging-related disorders and pathological processes, such as Alzheimer's disease.

\section{Acknowledgment}

The authors would like to thank Mr S. Josephs for his assistance in the acquisition of the MRI data, and IT Research (TCD) for providing access to computer resources.

\section{Author Disclosure Statement}

This study was part funded by the EU European Regional Development Fund 20072013 (Project 087 - Neuroskill) awarded to ALWB, PM, JM. FS was supported by a PhD Studentship awarded by Trinity College Dublin. No competing financial interests exist. 


\section{References}

Abdul-Rahman, M.F., Qiu, A., Sim, K. 2011. Regionally specific white matter disruptions of fornix and cingulum in schizophrenia. PloS one 6(4), e18652. doi:10.1371/journal.pone.0018652.

Beck AT., Steer RA., Ball R., Ranieri W. Comparison of Beck Depression Inventories -IA and -II in psychiatric outpatients. J Pers Assess, 1996 Dec;67(3):588-597.

Beckmann, M., Johansen-Berg, H., Rushworth, M.F. 2009. Connectivity-based parcellation of human cingulate cortex and its relation to functional specialization. The Journal of neuroscience : the official journal of the Society for Neuroscience 29(4), 1175-90. doi:10.1523/jneurosci.3328-08.2009.

Benjamini Y. and Hochberg Y. Journal of the Royal Statistical Society. Series B (Methodological), Vol. 57, No.1 (1995), 289-300.

Burdette, J.H., Durden, D.D., Elster, A.D., Yen, Y.F. 2001. High b-value diffusionweighted $\mathrm{MRI}$ of normal brain. Journal of computer assisted tomography 25(4), 515-9.

Cao, W., Luo, C., Zhu, B., Zhang, D., Dong, L., Gong, J., Gong, D., He, H., Tu, S., Yin, W., Li, J., Chen, H., Yao, D. 2014. Resting-state functional connectivity in anterior cingulate cortex in normal aging. Frontiers in aging neuroscience 6 , 280. doi:10.3389/fnagi.2014.00280.

Catheline, G., Periot, O., Amirault, M., Braun, M., Dartigues, J.F., Auriacombe, S., Allard, M. 2010. Distinctive alterations of the cingulum bundle during aging and Alzheimer's disease. Neurobiology of aging 31(9), 1582-92. doi:10.1016/j.neurobiolaging.2008.08.012.

Colby, J.B., Soderberg, L., Lebel, C., Dinov, I.D., Thompson, P.M., Sowell, E.R. 2012. Along-tract statistics allow for enhanced tractography analysis. Neurolmage 59(4), 3227-42. doi:10.1016/j.neuroimage.2011.11.004.

Folstein, M.F., Folstein, S.E., McHugh, P.R. 1975. "Mini-mental state". A practical method for grading the cognitive state of patients for the clinician. Journal of psychiatric research 12(3), 189-98.

Gunbey, H.P., Ercan, K., Findikoglu, A.S., Bulut, H.T., Karaoglanoglu, M., Arslan, H. 2014. The limbic degradation of aging brain: a quantitative analysis with diffusion tensor imaging. TheScientificWorldJournal 2014, 196513. doi:10.1155/2014/196513.

Head, D., Buckner, R.L., Shimony, J.S., Williams, L.E., Akbudak, E., Conturo, T.E., McAvoy, M., Morris, J.C., Snyder, A.Z. 2004. Differential vulnerability of anterior white matter in nondemented aging with minimal acceleration in dementia of the Alzheimer type: evidence from diffusion tensor imaging. Cerebral cortex (New York, NY : 1991) 14(4), 410-23.

Heilbronner, S.R., Haber, S.N. 2014. Frontal cortical and subcortical projections provide a basis for segmenting the cingulum bundle: implications for neuroimaging and psychiatric disorders. The Journal of neuroscience : the official journal of the Society for Neuroscience 34(30), 10041-54. doi:10.1523/jneurosci.5459-13.2014.

Jang, S.H., Kwon, Y.H., Lee, M.Y., Kim, J.R., Seo, J.P. 2016. Aging of the cingulum in the human brain: Preliminary study of a diffusion tensor imaging study. Neuroscience letters 610, 213-7. doi:10.1016/j.neulet.2015.11.018.

Jeurissen, B., Leemans, A., Jones, D.K., Tournier, J.D., Sijbers, J. 2011. Probabilistic fiber tracking using the residual bootstrap with constrained 
spherical deconvolution. Human brain mapping 32(3), 461-79. doi:10.1002/hbm.21032.

Jones, D.K., Christiansen, K.F., Chapman, R.J., Aggleton, J.P. 2013. Distinct subdivisions of the cingulum bundle revealed by diffusion MRI fibre tracking: implications for neuropsychological investigations. Neuropsychologia 51(1), 67-78. doi:10.1016/j.neuropsychologia.2012.11.018.

Lebel, C., Walker, L., Leemans, A., Phillips, L., Beaulieu, C. 2008. Microstructural maturation of the human brain from childhood to adulthood. Neurolmage 40(3), 1044-55. doi:10.1016/j.neuroimage.2007.12.053.

Leemans A, Jeurissen B, Sijbers J, and Jones DK. ExploreDTI: a graphical toolbox for processing, analyzing, and visualizing diffusion MR data. In: 17th Annual Meeting of Intl Soc Mag Reson Med, p. 3537, Hawaii, USA, 2009.

Leemans, A., Jones, D.K. 2009. The B-matrix must be rotated when correcting for subject motion in DTI data. Magnetic resonance in medicine 61(6), 1336-49. doi:10.1002/mrm.21890.

Madden, D.J., Bennett, I.J., Burzynska, A., Potter, G.G., Chen, N.K., Song, A.W. 2012. Diffusion tensor imaging of cerebral white matter integrity in cognitive aging. Biochimica et biophysica acta 1822(3), 386-400. doi:10.1016/j.bbadis.2011.08.003.

Morris, J.C., Mohs, R.C., Rogers, H., Fillenbaum, G., Heyman, A. 1988. Consortium to establish a registry for Alzheimer's disease (CERAD) clinical and neuropsychological assessment of Alzheimer's disease. Psychopharmacology bulletin 24(4), 641-52.

Nelson, Hazel E. (1982). The National Adult Reading Test (NART): test manual. Windsor: NFER-Nelson.

Nichols, T., Hayasaka, S. 2003. Controlling the familywise error rate in functional neuroimaging: a comparative review. Statistical methods in medical research 12(5), 419-46.

Park, D.C., Reuter-Lorenz, P. 2009. The adaptive brain: aging and neurocognitive scaffolding. Annual review of psychology 60, 173-96. doi:10.1146/annurev.psych.59.103006.093656.

Peters, R. 2006. Ageing and the brain. Postgraduate medical journal 82(964), 84-8. doi:10.1136/pgmj.2005.036665.

Pfefferbaum, A., Adalsteinsson, E., Sullivan, E.V. 2005. Frontal circuitry degradation marks healthy adult aging: Evidence from diffusion tensor imaging. Neurolmage 26(3), 891-9. doi:10.1016/j.neuroimage.2005.02.034.

Pievani, M., Agosta, F., Pagani, E., Canu, E., Sala, S., Absinta, M., Geroldi, C., Ganzola, R., Frisoni, G.B., Filippi, M. 2010. Assessment of white matter tract damage in mild cognitive impairment and Alzheimer's disease. Human brain mapping 31(12), 1862-75. doi:10.1002/hbm.20978.

Pruessmann, K.P., Weiger, M., Scheidegger, M.B., Boesiger, P. 1999. SENSE: sensitivity encoding for fast MRI. Magnetic resonance in medicine 42(5), 95262.

Reijmer, Y.D., Leemans, A., Heringa, S.M., Wielaard, I., Jeurissen, B., Koek, H.L., Biessels, G.J. 2012. Improved sensitivity to cerebral white matter abnormalities in Alzheimer's disease with spherical deconvolution based tractography. PloS one 7(8), e44074. doi:10.1371/journal.pone.0044074.

Sahakian, B.J., Morris, R.G., Evenden, J.L., Heald, A., Levy, R., Philpot, M., Robbins, T.W. 1988. A comparative study of visuospatial memory and 
learning in Alzheimer-type dementia and Parkinson's disease. Brain : a journal of neurology 111 ( Pt 3), 695-718.

Stadlbauer, A., Salomonowitz, E., Strunk, G., Hammen, T., Ganslandt, O. 2008. Quantitative diffusion tensor fiber tracking of age-related changes in the limbic system. European radiology 18(1), 130-7. doi:10.1007/s00330-007-0733-8.

Sullivan, E.V., Pfefferbaum, A. 2006. Diffusion tensor imaging and aging. Neuroscience and biobehavioral reviews 30(6), 749-61. doi:10.1016/j.neubiorev.2006.06.002.

Tournier, J.D., Calamante, F., Gadian, D.G., Connelly, A. 2004. Direct estimation of the fiber orientation density function from diffusion-weighted MRI data using spherical deconvolution. Neurolmage 23(3), 1176-85. doi:10.1016/j.neuroimage.2004.07.037. 
TABLES AND FIGURES

\begin{tabular}{|c|c|c|}
\hline Group & Young adults & Older adults \\
\hline Gender $M$ & 16 & 17 \\
\hline$F$ & 19 & 16 \\
\hline Age(yrs) & $22.3 \pm 2.7$ & $69.5 \pm 3.5$ \\
\hline NART & $117.27 \pm 4.7$ & $114.5 \pm 7.8$ \\
\hline $\begin{array}{l}\text { Education } \\
\text { (n. of yrs) }\end{array}$ & $17.1 \pm 2$ & $13.8 \pm 3.7$ \\
\hline MMSE & $\mathrm{n} / \mathrm{a}$ & $28.8 \pm 0.9$ \\
\hline
\end{tabular}

Table 1: Demographic information of the participants 


\begin{tabular}{l|llllll}
\hline CANTAB & $\begin{array}{l}\text { PRM initial } \\
\text { standard }\end{array}$ & $\begin{array}{l}\text { PAL total } \\
\text { errors } \\
\text { standard }\end{array}$ & $\begin{array}{l}\text { PAL t.e. 6 } \\
\text { shapes } \\
\text { standard }\end{array}$ & $\begin{array}{l}\text { SWM } \\
\text { between } \\
\text { errors } \\
\text { standard }\end{array}$ & $\begin{array}{l}\text { SWM } \\
\text { strategy } \\
\text { standard }\end{array}$ & $\begin{array}{l}\text { PRM- } \\
\text { delayed } \\
\text { standard }\end{array}$ \\
\hline Young & $0.99 \pm 0.58$ & $0.05 \pm 1.13$ & $0.03 \pm 1.0$ & $-0.02 \pm 0.9$ & $0.3 \pm 1.2$ & $-0.3 \pm 1.4$ \\
\hline Old & $0.7 \pm 0.9$ & $0.04 \pm 1.15$ & $0.1 \pm 0.9$ & $0.21 \pm 0.8$ & $-0.07 \pm 0.9$ & $-1.5 \pm 1.3$ \\
\hline
\end{tabular}

Table 2: Standardized results from CANTAB test battery

\begin{tabular}{|l|l|l|l|l|l|}
\hline \multirow{2}{*}{$\begin{array}{l}\text { Singulum } \\
\text { segment }\end{array}$} & \multirow{2}{*}{ Hemisphere } & \multicolumn{4}{l|}{ Diffusivity measures } \\
\cline { 3 - 6 } & & FA & MD & AD & RD \\
\hline Subgenual & Left & $0.006 \downarrow$ & ns & $0.014 \downarrow$ & $0.002 \uparrow$ \\
\hline & Right & ns & ns & ns & ns \\
\hline Retrosplenial & Left & ns & ns & ns & ns \\
\hline & Right & ns & ns & ns & ns \\
\hline Parahippocampal & Left & ns & ns & ns & ns \\
\hline & Right & ns & ns & ns & ns \\
\hline
\end{tabular}

Table 3: Between-group effect in the repeated-measures ANOVA 

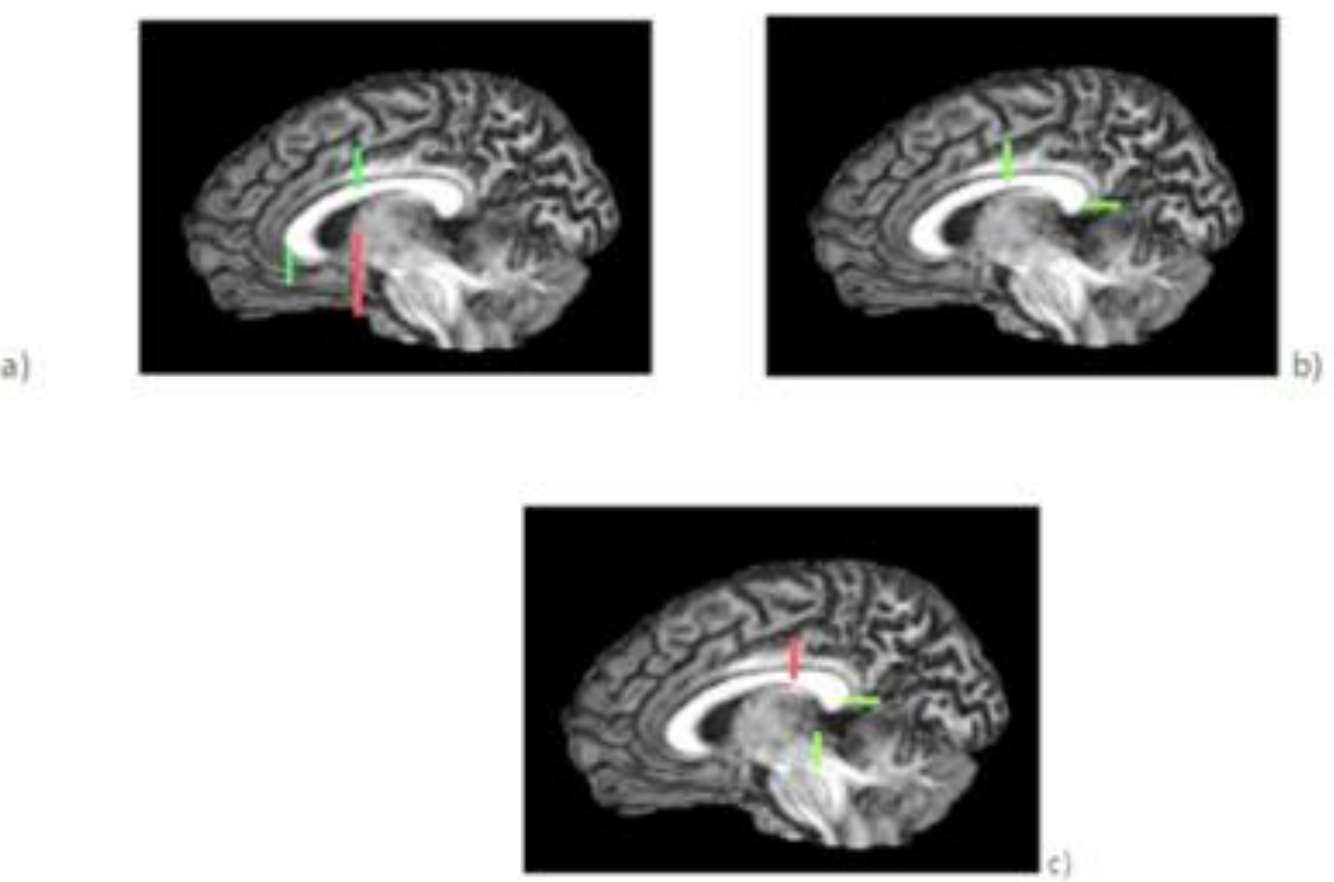

d)
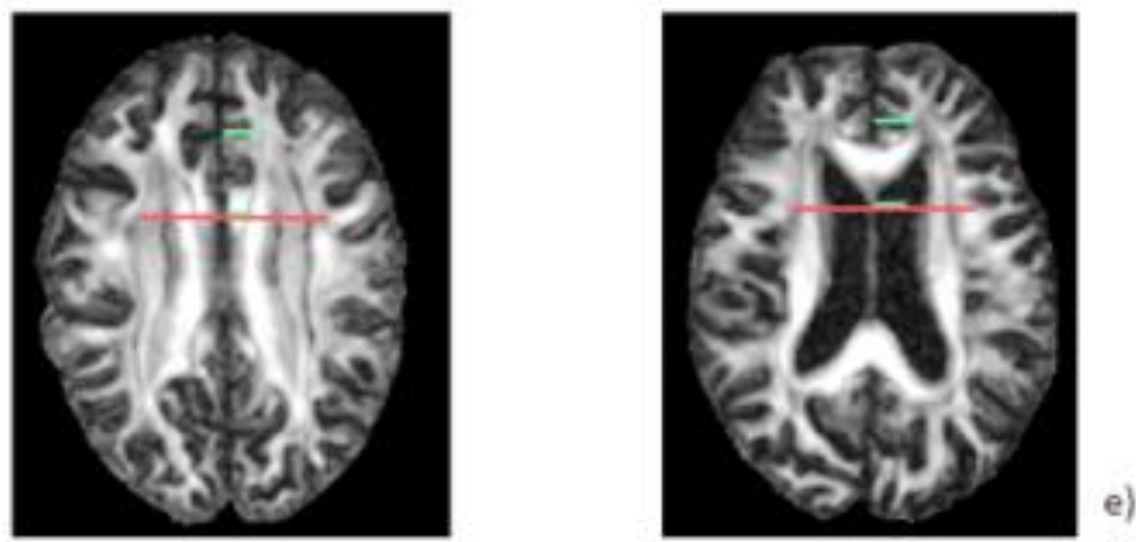

Figure 1: Definition of each cingulum segment: a) subgenual, b) retrosplenial and c) parahippocampal branches, sagittal view. Figures d) and e) show the location of ROls on d) a young subject and e) an old healthy subject. The ventricular enlargement made impossible to use the same template for both groups in the ABT approach. 

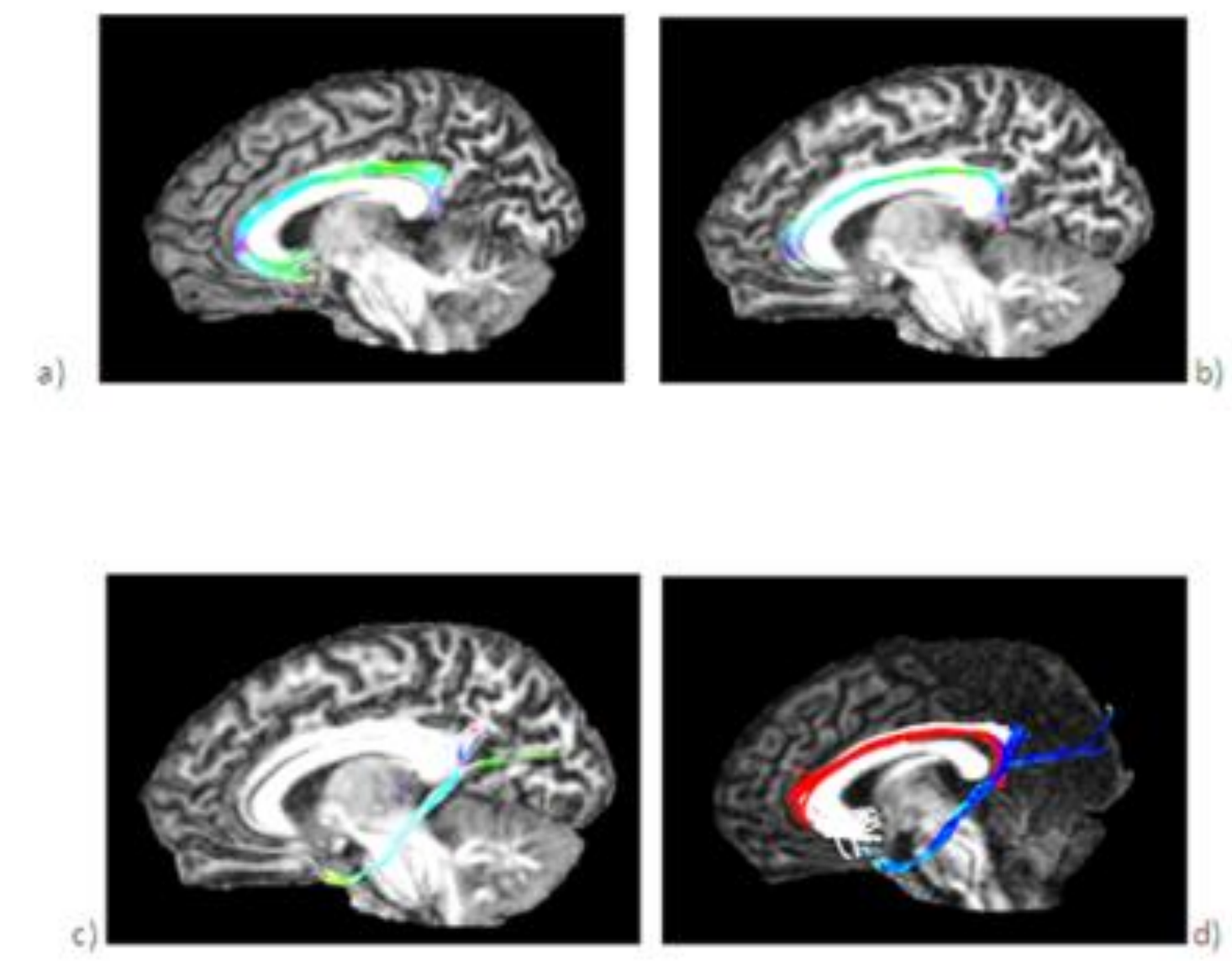

Figure 2: Output of the three different branches of the cingulum after ABT tractography. a) subgenual, b) retrosplenial and c) parahippocampal segment. d) the three segments with different colors (subgenual = white; retrosplenial = red; parahippocampal $=$ blue $)$. 

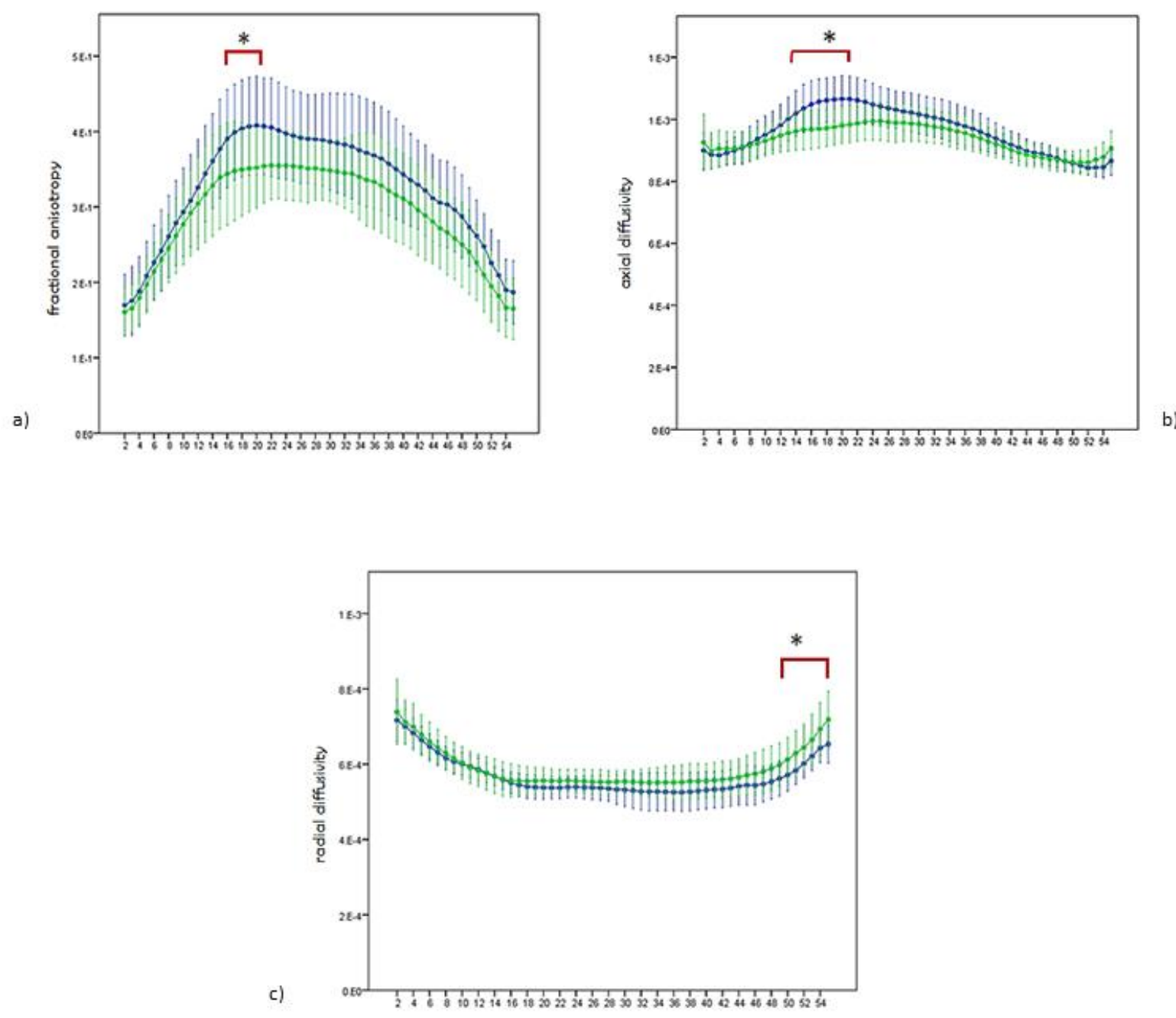

Figure 3: Along-tract analysis in the left hemisphere of the subgenual branch between young healthy people (blue line) and older healthy people (green line). The data represent the mean of DTI-measures for each point along the tract $+/-1$ 